\title{
Shear Wave Elastography Reveals a High Prevalence of NAFLD- related Fibrosis even in Type 1 Diabetes
}

\author{
Authors \\ Gesine Meyer $^{1}{ }^{*}$, Nina Dauth $^{1}{ }^{*}$, Matthias Grimm¹, Eva Herrmann², Joerg Bojunga1, Mireen Friedrich-Rust ${ }^{1}$
}

Affiliations

1 Department of Internal Medicine, Goethe-University Hospital, Frankfurt, Germany

2 Institute of Biostatistics and Mathematic Modelling, Goethe-University, Frankfurt, Germany

Key words

Diabetes mellitus, type 1, diabetes mellitus, type 2, elasticity imaging techniques, non-alcoholic fatty liver disease

$\begin{array}{ll}\text { received } & 22.07 .2021 \\ \text { revised } & 14.09 .2021 \\ \text { accepted } & 06.10 .2021\end{array}$

\section{Bibliography}

Exp Clin Endocrinol Diabetes 2022; 130: 532-538

DOI 10.1055/a-1666-0431

ISSN $0947-7349$

(C) 2021. Thieme. All rights reserved. Georg Thieme Verlag, Rüdigerstraße 14, 70469 Stuttgart, Germany

\section{Correspondence}

PD Dr. med. Gesine Meyer

Department of Internal Medicine 1

Division of Endocrinology and Diabetes

Goethe-University Hospital Frankfurt

Theodor-Stern-Kai 7

Frankfurt D-60590

Germany

Tel.: +49696301 5396, Fax: + 496963016405

gesine.meyer@kgu.de

\section{ABSTRACT}

Background The association between type 2 diabetes mellitus (T2DM) and advanced stages of non-alcoholic fatty liver disease is well known. Some studies indicate a relevant prevalence also in type 1 diabetes mellitus (T1DM), but so far there is only limited data.

Objective To determine the prevalence of non-alcoholic fatty liver disease (NAFLD)-related liver fibrosis in individuals with T1DM and compare to those with type 2 diabetes.

Methods Diabetic patients from a single diabetes care centre were screened for liver fibrosis by sonographic shear wave elastography (SWE). In addition, all patients received laboratory evaluation including non-alcoholic fatty liver fibrosis score and Fibrosis-4 Index.

Results Three hundred and forty patients were included in the study, of these, 310 received SWE. Overall 254 patients (93 with type 1 and 161 with type 2 diabetes) had reliable measurements and were included in the final analysis. In patients with type 1 diabetes, the prevalence of NAFLD-related liver fibrosis was $16-21 \%$, depending on the method of detection. Significant liver fibrosis was observed in $30-46 \%$ of patients with type 2 diabetes.

Conclusions Our data revealed an unexpectedly high prevalence of NAFLD-related liver fibrosis in patients with type 1 diabetes. To our knowledge, this is one of the first studies using SWE to diagnose advanced NAFLD in type 1 diabetes in a nonpreselected cohort. Considering the findings of our study, regular screening for hepatic complications must be recommended for all diabetic patients, even for those with type 1 diabetes.

\section{Introduction}

Non-alcoholic fatty liver disease (NAFLD) is the most common liver disease with an increasing prevalence worldwide affecting up to $40 \%$ of the population in Western countries [1-3]. NAFLD comprises a broad spectrum of severity including isolated hepatocellular accumulation of fat (steatosis), inflammatory processes resulting in hepatocyte injury (steatohepatitis) as well as progressive fibrosis that can lead to cirrhosis of liver tissue and the development of

Equal contribution hepatocellular carcinoma in some individuals [4, 5]. Association between NAFLD and obesity, dyslipidaemia, glucose intolerance, insulin resistance as well as type 2 diabetes mellitus (T2DM) are well known and proven by many studies [6,7]. Various genetic alleles increase the risk for NAFLD. Remarkably, the strongest of these risk alleles (148Met in PNPLA3 and 167Lys in TM6SF2) are associated with a lower risk of cardiovascular disease [8]. Usually, NAFLD and metabolic syndrome are associated bidirectionally [9]. Furthermore, pre-existing T2DM is an independent risk factor for a progression of NAFLD to non-alcoholic steatohepatitis (NASH) and 
finally fibrosis and cirrhosis in some cases [10-12]. Insulin resistance induces storage of fat in hepatocytes and leads to oxidative stress, worsening hepatic inflammation. Direct glucotoxicity to hepatocytes is suspected to be another trigger [11].

Liver biopsy and histopathological evaluation is still the gold standard for the diagnosis of NAFLD and, in particular, grades of inflammation, hepatocyte injury and fibrosis. However, since this method is invasive and the prevalence of NAFLD is high, there is a need for alternative, non-invasive methods [13]. B-mode liver ultrasound is a cost-effective and widely available method for the diagnosis of NAFLD but with only limited sensitivity; steatosis cannot be ascertained before fatty degeneration comprises a certain proportion of liver tissue. The method of B-mode liver ultrasound depends on the examiner's experience and assessment and cannot distinguish between fatty degeneration, steatohepatitis and fibrotic remodelling. Liver MRI is much more sensitive for the detection of steatosis, but not inflammation and fibrosis, and is limited by costs and availability. Laboratory findings, in particular, alanine aminotransferase (ALT) and aspartate aminotransferase (AST) are not helpful in the diagnosis of NAFLD or to discriminate the degree of liver injury [14]. Declining levels of albumin and coagulation factors as well as increasing bilirubin levels are signs of an advanced liver failure and inappropriate for NAFLD screening [15]. To screen patients for NAFLD, the NAFLD fibrosis score (NAFLD-FS) was developed. NAFLD-FS is determined by analysing routinely measured and readily available clinical and laboratory data (age, height, weight, presence of diabetes mellitus, AST, ALT, platelet count and serum albumin). It enables a non-invasive diagnosis of NAFLD with significant fibrosis as well as an exclusion of NAFLD-related fibrosis and is well validated [16]. However, by applying this scoring system, a relevant number of cases obtain an indeterminate result requiring further diagnostic steps.

Stiffness of liver tissue can be measured and quantified using sonographic shear wave elastography (SWE), a non-invasive and costeffective method to diagnose NAFLD-related fibrosis [17, 18]. The validity of this method could be confirmed in patients with diabetes [19]. A recent meta-analysis revealed a prevalence of NAFLD at $56 \%$ and $\mathrm{NASH}$ at $37 \%$ in patients with T2DM [20]. When transient elastography was used to diagnose significant NAFLD-related fibrosis in T2DM, the prevalence ranged between 17 and more than 20\% [20-23]. Several studies have indicated a relevant prevalence of NAFLD also in patients with type 1 diabetes mellitus (T1DM), but results are very heterogeneous. Depending on the diagnostic method, estimated prevalence varies between 9 and more than 70\% [24-27]. There are only limited data on the prevalence of NAFLD-related fibrosis in T1DM, in the range of about 2 to $20 \%$ [26, 28-30]. Transient elastography was so far used only in a few cases in these studies, indicating a prevalence of $2.1 \%$ [29] and $11 \%$ in a subgroup at particular risk [28]. None of these studies used liver biopsy results as a reference.

Our objective was to determine the prevalence of NAFLD-related fibrosis using SWE in patients with type 1 diabetes and compared it to those with type 2 diabetes.

\section{Subjects, Material and Methods}

The study was performed in accordance with the ethical guidelines of the Helsinki Declaration and approved by the local ethics com- mittee. It is registered at clinicaltrials.gov (NCT01997424). Assuming a prevalence of steatosis at about $60 \%$ and a prevalence of liver fibrosis at about $15 \%$ in individuals with T2DM [31], a sample size of $n=340$ was calculated to reach a confidence interval $(\mathrm{Cl})$ length of $8-10 \%$. All patients presenting to our in- and outpatient diabetes care centre were evaluated for inclusion in the study. Informed consent was obtained from all participants. The study was conducted from 2016 to 2019. Inclusion criteria were the presence of T1or T2DM and a minimum age of 18 years. Exclusion criteria were hazardous alcohol consumption (>14 standard units/week in women, respectively $>21$ standard units/week in men), pre-existing chronic liver disease (viral or autoimmune hepatitis, hemochromatosis, Wilson's disease, primary biliary cirrhosis and primary sclerosing cholangitis), pre-existing hepatocellular carcinoma or liver metastases, ascites, pregnancy and lactation period. Data about the duration of DM, diabetic complications, pre-existing liver diseases, further concomitant disorders and alcohol consumption were collected from medical files and by interview. Blood tests for liver enzymes (ALT, AST and GGT), HbA1c, blood count and serum lipids (total cholesterol, high-density lipoprotein cholesterol (HDLC), low-density lipoprotein cholesterol (LDL-C) and triglycerides (TG)) were estimated under fasting conditions. Height, weight and waist circumference were measured using calibrated devices.

NAFLD-FS as well as Fibrosis-4 (FIB-4) Index were calculated for every patient using earlier published formulae [16, 32]. Cut-off values for significant fibrosis have been defined as a NAFLD-FS >0.676 (positive predictive value $>90 \%$ ), and for exclusion of fibrosis $<-1.455$ (negative predictive value $>93 \%$ ) [16-33]. For FIB-4, cut-off levels for significant fibrosis were defined as a score $>3.25$ (positive predictive value $65 \%$, specificity $97 \%$ ), and $<1.3$ (negative predictive value $90 \%$, sensitivity $70 \%$ ) for exclusion of significant fibrosis [34].

All participants underwent an ultrasound of the liver, followed by sonographic SWE, which is a method to evaluate the elastic properties of tissues while performing real-time $B$-mode imaging. The tissue within a region-of-interest (ROI) cursor is mechanically excited using short-duration ( $262 \mu \mathrm{s}$ ) acoustic pulses which generate localized tissue displacements. These displacements induce a lateral shear-wave propagation, tracked by multiple laterally positioned ultrasound tracking beams. Investigations were performed using pointSWE (Siemens Acuson 2000 or Hitachi Ascendus, Hitachi Medical Corporation, Tokyo, Japan) or 2D-SWE (Toshiba Aplio 500, Toshiba Medical Systems Corporation, Tochigi, Japan)). The shear wave velocity (SWV) within the tissue was reconstructed using acoustic radiation force imaging (ARFI) (Siemens Acuson 2000), by measuring the time to peak displacement at each lateral location. The shear wave propagation velocity is proportional to the square root of tissue elasticity [35]. The stiffer is the tissue the faster is the spread of the shear wave. Results are expressed in $\mathrm{m} / \mathrm{s}$ in a measurement range of $0.5-4.9 \mathrm{~m} / \mathrm{s}$. For classification of the degree of fibrosis, in accordance with the recommendations by Siemens the following cut-off values were applied: for the diagnosis of significant fibrosis (F2), $1.34 \mathrm{~m} / \mathrm{s}$; for the diagnosis of severe fibrosis (F3), $1.55 \mathrm{~m} / \mathrm{s}$; for the diagnosis of liver cirrhosis (F4), $1.80 \mathrm{~m} / \mathrm{s}$ [36]. For estimations using Hitachi Ascendus and Toshiba Aplio 500 systems, tissue elasticity was expressed as tensile modulus in $\mathrm{kPa}$. For classification of the degree of fibrosis, the following cut-off values were applied in accordance with the recommendations by the respective 
companies: for the diagnosis of significant fibrosis (F2) $7.0 \mathrm{kPa}$ (Toshiba Aplio 500) and $7.1 \mathrm{kPa}$ (Hitachi Ascendus), for the diagnosis of severe fibrosis (F3) $7.95 \mathrm{kPa}$ (Toshiba Aplio 500) and $9.5 \mathrm{kPa}$ (Hitachi Ascendus) and the diagnosis of liver cirrhosis (F4) $10.5 \mathrm{kPa}$ (Toshiba Aplio 500 ) and $12.5 \mathrm{kPa}$ (Hitachi Ascendus). Examinations were carried out under fasting conditions and at baseline breathing. Five measurements were performed in each subject and median SWV respectively tensile modulus was calculated for further analyses. SWE measurements were defined as reliable if the interquartile range was $\leq 30 \%$. Since ARFI was the only SWE method used in this study, which is directly validated by liver biopsy and histopathological evaluation as reference standard methods to diagnose NAFLD and stage of fibrosis [17], a subgroup analysis of patients examined through this method was performed ( $n=137$; thereof $n=88$ with T2DM and $n=49$ with T1DM).

Clinical and laboratory characteristics were expressed as median and interquartile range and $\mathrm{n}(\%)$. Since dimensional units as well as cut-off values for degree of fibrosis differ between the different methods of SWE (Siemens Acuson 2000 ARFI, Hitachi Ascensus and Toshiba Aplio 500), a direct analysis of measurement values was not applicable. Therefore, SWE results were classified in three clinically relevant grades of fibrosis (no significant fibrosis: F1, significant fibrosis: F2-F4 and severe fibrosis: F3-F4). Correlations between possible influencing factors (age, sex, duration of diabetes, BMI, waist circumference, HbA1c and blood lipids) and grade of fibrosis were calculated using contingency tables and proofed for independence using the Chi-square test and Fisher `s exact test, respectively. Continuous potential predictors (age and BMI) were analysed by logistic regression. Statistical analysis was performed using the statistical software BiAS 11.06 (Epsilon 2017, Frankfurt am Main, Germany). Statistical significance was accepted for $P$ values of less than 0.05 .

\section{Results}

Three hundred forty patients were screened and a complete data set including laboratory results and SWE could be collected for $n=310$ participants ( $n=197$ with T2DM and $n=113$ with T1DM).
In $n=161$ (82\%) patients with T2DM and $n=93$ (82\%) with T1DM, a reliable SWE measurement could be performed. These individuals were included in the final analysis ( $\vee$ Fig. 1). Characteristics of included patients are summarized in > Table 1.

\section{Type 1 diabetes mellitus}

B-mode liver ultrasound could detect the prevalence of hepatic steatosis in $35.4 \%$ of patients with T1DM. The finding of hepatic steatosis using B-mode liver ultrasound and hepatic fibrosis using transient elastography were not significantly associated $(C C=0.02 ; \mathrm{p}=0.92)$.

Significant liver fibrosis was observed in $21 \%$ of the participants. Approximately half of them fulfilled the criterion for severe fibrosis. Calculating NAFLD-FS, significant fibrosis was assumed in $5.4 \%$, and $2.2 \%$ using FIB-4 ( $\triangleright$ Table 2). NAFLD-FS and FIB-4 excluded fibrosis in, respectively, in 43 and $72 \%$ of the patients.

Serum triglycerides were significantly positively (OR 1.01, $95 \%$ $\mathrm{Cl} 1.00-1.02 ; \mathrm{p}=0.03$ ) and high-density lipoprotein cholesterol levels significantly negatively (OR 0.97, 95\% Cl 0.94-0.99; $p=0.04$ ) correlated with the grade of fibrosis, whereas age, sex, BMI, waist and hip circumference, waist-hip-ratio, duration of diabetes as well as HbA1c showed no significant correlation ( $>$ Table 3 ). Neither NAFLD-FS nor FIB-4 was significantly correlated with the finding of significant liver fibrosis determined by SWE (NAFLD-FS CC $=0$, $\mathrm{p}=1.0$; FIB-4 CC $=0.02, \mathrm{p}=0.4$ ).

Using ARFI, significant liver fibrosis was detected in $16 \%$ of the patients with T1DM. The criteria for severe fibrosis were fulfilled by $10 \%$ of the patients. Calculating NAFLD-FS in this subgroup, significant fibrosis was assumed in 4.1 and $2.0 \%$ using FIB-4 ( $\triangleright$ Table 2) A significant fibrosis was excluded in $43 \%$ using NAFLD-FS and in $74 \%$ using FIB-4. In this subgroup, there were no significant correlations with age, sex, BMI, waist circumference, HbA1c, duration of diabetes and blood lipids. FIB-4 was again not significantly correlated with the finding of significant liver fibrosis determined by SWE in this subgroup $(C C=0.21, p=0.1)$, while paradoxically, NAFLD-FS showed a significant inverse correlation $(C C=-0.55$, $\mathrm{p}=0.04)$.

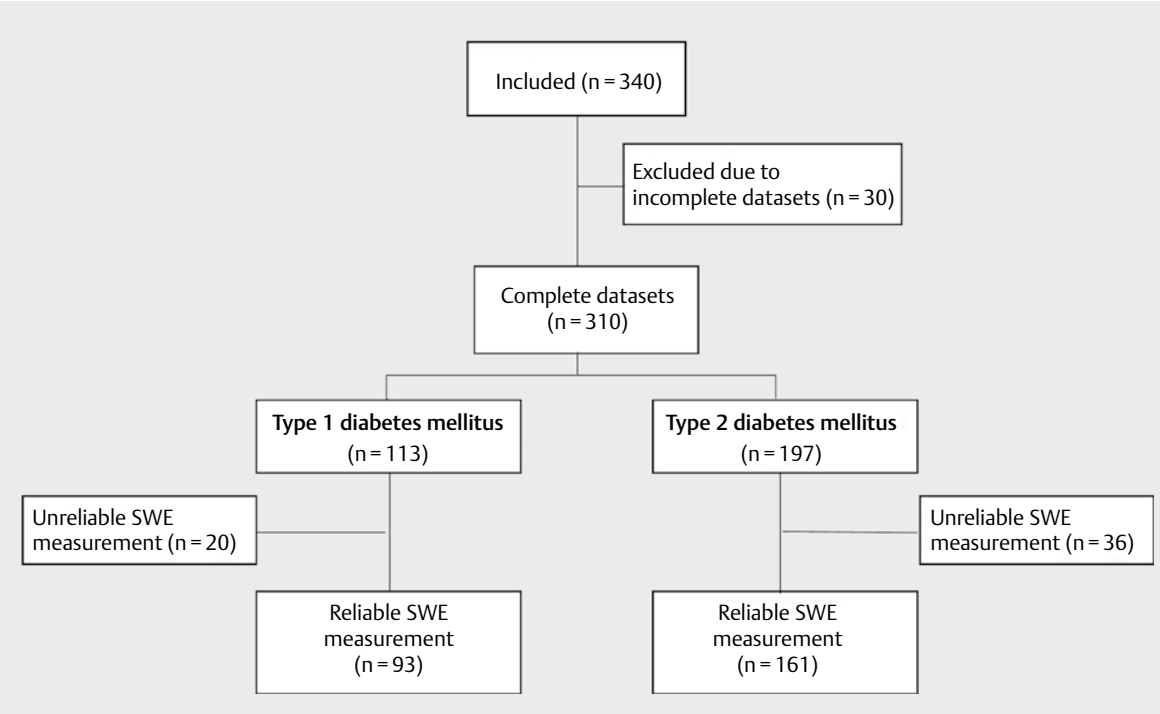

Fig. 1 The CONSORT diagram. 
- Table 1 Baseline characteristics of participants with either type 1 or type 2 diabetes mellitus included in the final data analysis, differentiated in the entire group as well as the subgroups examined using Acoustic Radiation Force Imaging (ARFI).

\begin{tabular}{|c|c|c|c|c|c|c|}
\hline & \multicolumn{3}{|c|}{ Type 1 diabetes mellitus $(n=93)$} & \multicolumn{3}{|c|}{ Type 2 diabetes mellitus $(n=161)$} \\
\hline & $\begin{array}{l}\text { entire group } \\
(n=93)\end{array}$ & $\begin{array}{l}\text { ARFI } \\
(n=49)\end{array}$ & $\begin{array}{l}\text { no ARFI } \\
(n=44)\end{array}$ & $\begin{array}{l}\text { entire group } \\
(n=161)\end{array}$ & $\begin{array}{l}\text { ARFI } \\
(n=88)\end{array}$ & $\begin{array}{l}\text { no ARFI } \\
(n=73)\end{array}$ \\
\hline Men & $51(55)$ & $33(67)$ & $18(41)$ & $93(58)$ & $53(60)$ & $40(55)$ \\
\hline Age (years) & $55(41-70)$ & $53(44-67)$ & $49(29-66)$ & $63(53-73)$ & $65(56-76)$ & $63(53-73)$ \\
\hline Duration of diabetes (years) & $28(15-40)$ & $29(17-43)$ & $25(10-40)$ & $12(6-20)$ & $14(6-20)$ & $12(6-20)$ \\
\hline $\mathrm{BMI}\left(\mathrm{kg} / \mathrm{m}^{2}\right)$ & $26.3(23.4-29.9)$ & $25.3(22.1-29.1)$ & $25.9(23.2-28.6)$ & $31.4(27.1-35.3)$ & $29.6(26.3-33.6)$ & $31(27-35)$ \\
\hline Waist circumference $(\mathrm{cm})$ & $96(83-104)$ & $96(84-104)$ & $89(78-98)$ & $111(101-122)$ & $109(101-117)$ & $110(98-121)$ \\
\hline Hip circumference $(\mathrm{cm})$ & $100(94-108)$ & $100(85-110)$ & $98(99-105)$ & $110(100-123)$ & $108(97-118)$ & $111(105-120)$ \\
\hline Waist-hip ratio & $0.96(0.87-1.04)$ & $0.96(0.86-1.04)$ & $0.88(0.85-1.01)$ & $1.01(0.95-1.06)$ & $1.01(0.95-1.09)$ & $1(0.91-1.06)$ \\
\hline $\operatorname{HbA1c}(\%)$ & $7.1(6.6-7.6)$ & $7.4(6.8-8.0)$ & $6.9(6.4-7.2)$ & $7.1(6.4-8.0)$ & $7.4(6.5-8.2)$ & $7(6.4-7.9)$ \\
\hline Triglycerides (mmol/l) & $0.84(0.65-1.28)$ & $1.03(0.75-1.28)$ & $0,76(0.65-1.06)$ & $1.81(1.29-2.46)$ & $1.84(1.25-2.52)$ & $1.84(1.27-2.55)$ \\
\hline HDL-cholesterol (mmol/l) & $1.69(1.39-2.10)$ & $1.66(1.35-2.05)$ & $1.9(1.48-2.43)$ & $1.20(1.00-1.50)$ & $1.20(1.01-1.48)$ & $1.2(0.97-1.41)$ \\
\hline AST $(\mathrm{nmol} / \mathrm{l})$ & $367(316-442)$ & & & $400(333-533)$ & & \\
\hline ALT $(\mathrm{nmol} / \mathrm{l})$ & $333(267-417)$ & & & $417(308-617)$ & & \\
\hline GGT (nmol/l) & $300(200-392)$ & & & $500(350-850)$ & & \\
\hline albumin $(\mathrm{g} / \mathrm{l})$ & $44(42-46)$ & & & $43(41.5-45)$ & & \\
\hline \multicolumn{7}{|l|}{ Antidiabetic treatment } \\
\hline Biguanides & $3(2.8)$ & & & $103(64)$ & & \\
\hline DDP4 inhibitors & $1(0.93)$ & & & $58(36)$ & & \\
\hline GLP-1 receptor agonists & $2(1.86)$ & & & $45(28)$ & & \\
\hline SGLT-2 inhibitors & $0(0)$ & & & $29(18)$ & & \\
\hline Insulin & $93(100)$ & & & $105(65)$ & & \\
\hline \multicolumn{7}{|l|}{ Concomitant conditions } \\
\hline Cardiovascular disease & $13(14)$ & & & $48(30)$ & & \\
\hline Diabetic nephropathy & $16(17)$ & & & $60(37)$ & & \\
\hline Arterial hypertension & $52(56)$ & & & $137(85)$ & & \\
\hline
\end{tabular}

- Table 2 Prevalence of non-alcoholic fatty liver disease (NAFLD)-related liver fibrosis in patients with diabetes mellitus, as diagnosed by shear wave elastography (SWE), NAFLD fibrosis score (NAFLD-FS), Fibrosis-4 (FIB-4) Index and in a subgroup using Acoustic Radiation Force Imaging (ARFI).

\begin{tabular}{|c|c|c|c|c|c|}
\hline & & \multicolumn{2}{|c|}{ Type 1 diabetes mellitus } & \multicolumn{2}{|c|}{ Type 2 diabetes mellitus } \\
\hline & & $n=93$ & $\begin{array}{l}\text { Subgroup ARFI } \\
(\mathrm{n}=49)\end{array}$ & $n=161$ & $\begin{array}{l}\text { Subgroup ARFI } \\
(\mathrm{n}=\mathbf{8 8})\end{array}$ \\
\hline \multirow[t]{3}{*}{ No significant fibrosis } & SWE (grade F1) & $74(80)$ & $41(84)$ & $87(54)$ & $61(69)$ \\
\hline & NAFLD-FS $(<-1.455)$ & $40(43)$ & $21(43)$ & $33(21)$ & $16(18)$ \\
\hline & FIB-4 $(<1.3)$ & $67(72)$ & $36(74)$ & $86(53)$ & $42(48)$ \\
\hline \multirow[t]{2}{*}{ Possible fibrosis } & NAFLD-FS (-1.455-0.676) & $48(52)$ & $26(53)$ & $91(57)$ & $47(53)$ \\
\hline & FIB-4 (1.3-3.25) & $24(26)$ & $12(24)$ & $66(41)$ & $40(45)$ \\
\hline \multirow[t]{3}{*}{ Significant fibrosis } & SWE (grade F2-F4) & $19(21)$ & $8(16)$ & $74(46)$ & $27(31)$ \\
\hline & NAFLD-FS $(>0.676)$ & $5(5.4)$ & $2(4.1)$ & $37(23)$ & $25(28)$ \\
\hline & FIB-4 (>3.25) & $2(2.2)$ & $1(2.0)$ & $9(5.6)$ & $6(6.8)$ \\
\hline Severe fibrosis & SWE (grade F3-F4) & $11(12)$ & $5(10)$ & $39(24)$ & $19(22)$ \\
\hline
\end{tabular}

\section{Type 2 diabetes mellitus}

In nearly half ( $46 \%$ ) of the patients with T1DM, significant liver fibrosis (grade F2-F4) was detected using SWE. The criteria for severe fibrosis (F3-F4) were fulfilled by about one quarter (24\%) of those using SWE. Calculating NAFLD-FS, significant fibrosis was assumed in $23 \%$, but only in $5.6 \%$ using FIB-4. ( Table 2). Significant fibrosis could be excluded in 33 (21\%) individuals using NAFLD-FS and in 86 (53\%) using FIB-4. 
- Table 3 Odd ratios $(95 \% \mathrm{Cl}$ ) of factors associated with grade of fibrosis (no significant fibrosis; significant fibrosis; severe fibrosis) as diagnosed by shear wave elastography (SWE) from a univariable logistic regression in patients with type 1 and type 2 diabetes mellitus.

\begin{tabular}{|c|c|c|c|c|}
\hline \multirow[b]{2}{*}{ Age (years) } & \multicolumn{2}{|c|}{ Type 1 diabetes mellitus } & \multicolumn{2}{|c|}{ Type 2 diabetes mellitus } \\
\hline & $1.01(0.99,1.04)$ & $p=0.35$ & $1.00(0.97,1.02)$ & $p=0.80$ \\
\hline Sex & 0.05 & $p=0.73$ & 0.19 & $p=0.09$ \\
\hline Duration of diabetes (years) & $1.01(0.97,1.04)$ & $p=0.77$ & $0.99(0.96,1.02)$ & $p=0.59$ \\
\hline $\operatorname{BMI}\left(\mathrm{kg} / \mathrm{m}^{2}\right)$ & $1.02(0.90,1.16)$ & $\mathrm{p}=0.80$ & $1.09(1.03,1.15)$ & $p=0.003$ \\
\hline Waist circumference $(\mathrm{cm})$ & $1.00(0.96,1.06)$ & $\mathrm{p}=0.80$ & $1.03(1.00,1.07)$ & $p=0.09$ \\
\hline Hip circumference $(\mathrm{cm})$ & $0.98(0.92,1.05)$ & $p=0.6$ & $1.04(1.00,1.08)$ & $p=0.04$ \\
\hline Waist-hip ratio & $10.1(0.03,3758)$ & $p=0.4$ & $0.28(0.00,51.32)$ & $p=0.63$ \\
\hline $\mathrm{HbA1c}(\%)$ & $1.03(0.64,1.67)$ & $\mathrm{p}=0.89$ & $0.90(0.74,1.1)$ & $p=0.32$ \\
\hline Triglycerides (nmol/l) & $1.01(1.00,1.02)$ & $p=0.03$ & $1.00(0.99,1.00)$ & $p=0.40$ \\
\hline Total cholesterol (nmol/l) & $0.99(0.98,1.02)$ & $p=0.28$ & $0.90(0.99,1.01)$ & $P=0.60$ \\
\hline HDL-cholesterol (nmol/l) & $0.97(0.94,0.99)$ & $p=0.04$ & $1.01(0.98,1.03)$ & $p=0.68$ \\
\hline LDL-cholesterol (nmol/l) & $1.00(0.98,1.02)$ & $p=0.84$ & $0.90(0.99,1.01)$ & $p=0.50$ \\
\hline
\end{tabular}

Data are presented as odds ratio $(95 \% \mathrm{Cl})$, respectively contingency coefficient $c c$ for sex. BMI: Body mass index; HDL: High density lipoprotein; LDL: Low density lipoprotein.

Using the SWE criterion, a significant correlation was observed between BMI $\left(\mathrm{kg} / \mathrm{m}^{2}\right)$ and stage of fibrosis (OR 1.09, $95 \% \mathrm{Cl} 1.03-$ $1.15 ; p=0.003$ ). Furthermore, we could notice a significant, albeit marginal, positive correlation between the hip circumference and stage of fibrosis in patients with T2DM (OR 1.04, 95\% Cl 1.00-1.08; $p=0.04)$. Age, sex, waist circumference, waist-hip-ratio, HbA1c, duration of diabetes as well as blood lipids showed no significant correlation ( $>$ Table 3). Neither NAFLD-FS nor FIB-4 was significantly correlated with the finding of significant liver fibrosis determined by SWE (NAFLD-FS CC =0.07, p = 0.35; FIB-4 CC =0.05, p=0.50).

In the subgroup of patients with T2DM who were examined using ARFI, significant liver fibrosis was detected in $31 \%$ and the criteria for severe fibrosis were fulfilled by $22 \%$. Calculating NAFLDFS in this subgroup, significant fibrosis was assumed in $28 \%$ using NAFLD-FS and in $6.8 \%$ using FIB-4 ( $\triangleright$ Table 2). Significant fibrosis could be excluded by NAFLD-FS in $18 \%$ of the individuals and by FIB-4 in $48 \%$ cases. On comparing the overall cohort, only BMI was significantly correlated with the stage of fibrosis (OR 1.12, $95 \% \mathrm{Cl}$ $1.03-1.20 ; p=0.006)$. Even in this subgroup NAFLD-FS as well as FIB-4 were not significantly correlated with the finding of significant liver fibrosis determined by SWE (NAFLD-FS CC =0.06, $p=0.70$; FIB-4 CC $=0.2, p=0.17$ ).

\section{Discussion}

Our data reveal a remarkable prevalence of NAFLD-related liver fibrosis in T1DM in about one-fifth of the individuals examined. We, therefore, assume that NAFLD-related liver fibrosis is a previously underestimated complication in T1DM.

Regarding T2DM, our findings even exceed those of previous studies, showing significant NAFLD-related liver fibrosis in about a third of the subgroup examined with pSWE using ARFI.

The results of our study confirm the importance of screening for liver fibrosis not only in patients with type 2 but also those with type 1 diabetes to enable the detection of individuals at risk of developing hepatological sequelae.
In our cohort, unfavourable blood lipid levels were significantly correlated with the presence of fibrosis in T1DM, whereas other factors associated with metabolic syndrome were not. Concomitant metabolic pathologies seem to contribute, but do not sufficiently explain the development of liver fibrosis in T1DM. With the estimated median $\mathrm{HbA} 1 \mathrm{c}$ of $7.1 \%$, glycaemic control was very satisfactory in our cohort. This leaves the question, whether the prevalence of fibrosis could even be higher in individuals with worse glycaemic control. With a median of 28 years (IQR 15-40) duration of diabetes was noticeably long in our cohort. Presumably, exposure time of hepatocytes to glucose levels exceeding the physiological range is a contributing factor for fibrosis development. However, in our cohort of patients with long-standing T1DM, we could not verify a correlation between significant fibrosis and duration of disease. Further studies are necessary to understand pathogenesis and risk factors, especially in T1DM.

The limiting factors of our design were the application of three different methods of SWE. Nonetheless, this fact reflects clinical reality, particularly regarding the high prevalence of diabetes as well as NAFLD in the general population. To avoid the effect of different SWE methods, we performed a subgroup analysis of individuals examined with pointSWE using ARFI, which has been validated by liver biopsy and histological evaluation in previous studies [17]. In this subgroup, the prevalence of NAFLD-related fibrosis was lower in T1DM and in T2DM in comparison to the overall cohort, but still markedly higher than that indicated by previous data (16 vs. $2.1 \%$ in type 1 [29] and 31 vs. $18-24 \%$ in type 2 diabetes, respectively [21-23]). Baseline characteristics, notably for BMI, waist circumference, triglycerides as well as the duration of diabetes did not differ materially between our and these former examined cohorts. Age, as well as HbA1c, were tendentially even lower in these participants.

Unexpectedly, in our study, NAFLD-FS and FIB-4 were not significantly positively correlated with significant liver fibrosis detected by SWE, both in patients with T1DM or T2DM. The reasons remain unclear, in particular since NAFLD-FS is well evaluated at least in patients with T2DM [14]. Moreover, our study is possibly under- 
powered to address the question regarding the high prevalence of diabetes as well as NAFLD in western countries.

Considering that the results of our study revealing a high prevalence of NAFLD-related liver fibrosis not only in T2DM but also in T1DM, regular screening for hepatic complications should be implemented for all patients with diabetes. Since liver histology requires an invasive biopsy of liver tissue, this diagnostic gold standard is not justified for screening in all individuals with diabetes mellitus. SWE offers a non-invasive, cost-effective and sufficient opportunity for early recognition of diabetic liver involvement. Individuals affected require a thorough and regular hepatological surveillance for further complications.

\section{Funding}

This research did not receive any specific grant from funding agencies in the public, commercial, or not-for-profit sectors.

\section{Conflict of interest}

The authors declare that they have no conflicts of interest that could be perceived as prejudicing the impartiality of the research reported.

\section{References}

[1] Szczepaniak LS, Nurenberg P, Leonard D et al. Magnetic resonance spectroscopy to measure hepatic triglyceride content: Prevalence of hepatic steatosis in the general population. Am J Physiol - Endocrinol Metab 2005; 288: E462-E468

[2] Williams CD, Stengel J, Asike MI et al. Prevalence of nonalcoholic fatty liver disease and nonalcoholic steatohepatitis among a largely middle-aged population utilizing ultrasound and liver biopsy: A prospective study. Gastroenterology 2011; 140: 124-131

[3] Lee JY, Kim KM, Lee SG et al. Prevalence and risk factors of non-alcoholic fatty liver disease in potential living liver donors in Korea: $\mathrm{A}$ review of 589 consecutive liver biopsies in a single center. J Hepatol 2007; 47: 239-244

[4] Ekstedt M, Nasr P, Kechagias S. Natural history of NAFLD/NASH. Curr Hepatol Reports 2017; 16: 391-397

[5] Rinella ME. Nonalcoholic fatty liver disease A systematic review. JAMA 2015; 313: 2263-2273

[6] Meex RCR, Watt M]. Hepatokines: Linking nonalcoholic fatty liver disease and insulin resistance. Nature Rev Endocrinol 2017; 13: 509-520

[7] Lonardo A, Lugari S, Ballestri S.etal. A round trip from nonalcoholic fatty liver disease to diabetes: molecular targets to the rescue? Acta Diabetol 2019; 56: 385-396

[8] Stefan N, Häring HUH, Cusi K. Non-alcoholic fatty liver disease: Causes, diagnosis, cardiometabolic consequences, and treatment strategies. Lancet Diabetes Endocrinol 2019; 7: 313-324

[9] Lonardo A, Nascimbeni F, Mantovani A et al. Hypertension, diabetes, atherosclerosis and NASH: Cause or consequence? J Hepatol 2018; 335-352

[10] Adams LA, Lymp JF St., Sauver J et al. The natural history of nonalcoholic fatty liver disease: A population-based cohort study. Gastroenterology 2005; 129: 113-121
[11] Porepa L, Ray JG, Sanchez-Romeu P et al. Newly diagnosed diabetes mellitus as a risk factor for serious liver disease. CMAJ 2010; 182: E526-E531

[12] Tada T, Toyoda H, Sone $\mathrm{Y}$ et al. Type 2 diabetes mellitus: A risk factor for progression of liver fibrosis in middle-aged patients with non-alcoholic fatty liver disease. J Gastroenterol Hepatol 2019; 34: 2011-2018

[13] Castera L, Friedrich-Rust M, Loomba R. Noninvasive assessment of liver disease in patients with nonalcoholic fatty liver disease. Gastroenterology 2019; 156: 1264-1281

[14] Baršić N, Lerotić I, Smirčić-Duvnjak L et al. Overview and developments in noninvasive diagnosis of nonalcoholic fatty liver disease. World J Gastroenterol 2012; 18: 3945-3954

[15] Pandyarajan V, Gish RG, Alkhouri N et al. Screening for nonalcoholic fatty liver disease in the primary care clinic. Gastroenterol Hepatol 2019; 15: 357-365

[16] Angulo P, Hui JM, Marchesini G et al. The NAFLD fibrosis score: A noninvasive system that identifies liver fibrosis in patients with NAFLD. Hepatology 2007; 45: 846-854

[17] Cassinotto C, Boursier J, de Lédinghen V et al. Liver stiffness in nonalcoholic fatty liver disease: A comparison of supersonic shear imaging, FibroScan, and ARFI with liver biopsy. Hepatology 2016; 63: 1817-1827

[18] Liu H, Fu J, Hong R et al. Acoustic radiation force impulse elastography for the non-invasive evaluation of hepatic fibrosis in non-alcoholic fatty liver disease patients: A systematic review \& meta-analysis. PLoS One 2015; 10: e0127782

[19] Casey SP, Kemp WW, Mclean CA et al. A prospective evaluation of the role of transient elastography for the detection of hepatic fibrosis in type 2 diabetes without overt liver disease. Scand J Gastroenterol 2012; 47: 836-841

[20] Younossi ZM, Golabi P, de Avila L et al. The global epidemiology of NAFLD and NASH in patients with type 2 diabetes: A systematic review and meta-analysis. J Hepatol 2019; 71: 793-801

[21] Lombardi R, Airaghi L, Targher G et al. Liver fibrosis by FibroScan independently of established cardiovascular risk parameters associates with macrovascular and microvascular complications in patients with type 2 diabetes. Liver Int 2020; 40: 347-354

[22] Mantovani A, Turino T, Lando MG et al. Screening for non-alcoholic fatty liver disease using liver stiffness measurement and its association with chronic kidney disease and cardiovascular complications in patients with type 2 diabetes. Diabetes Metab 2020; 46: 296-303

[23] Mansour A, Mohajeri-Tehrani MR, Samadi M et al. Risk factors for non-alcoholic fatty liver disease-associated hepatic fibrosis in type 2 diabetes patients. Acta Diabetol 2019; 56: 1199-1207

[24] Cusi K, Sanyal AJ, Zhang $S$ et al. Non-alcoholic fatty liver disease (NAFLD) prevalence and its metabolic associations in patients with type 1 diabetes and type 2 diabetes. Diabetes, Obes Metab 2017; 19 : 1630-1634

[25] Serra-Planas E, Aguilera E, Castro L et al. Low prevalence of non-alcoholic fatty liver disease in patients with type 1 diabetes is associated with decreased subclinical cardiovascular disease. J Diabetes 2017; 9: 1065-1072

[26] Singh A, Le P, Lopez R et al. The utility of noninvasive scores in assessing the prevalence of nonalcoholic fatty liver disease and advanced fibrosis in type 1 diabetic patients. Hepatol Int 2018; 12 : 37-43

[27] Sviklāne L, Olmane E, Dzērve Z et al. Fatty liver index and hepatic steatosis index for prediction of non-alcoholic fatty liver disease in type 1 diabetes. J Gastroenterol Hepatol 2018; 33: 270-276 
[28] Marjot T, Sbardella E, Moolla A et al. Prevalence and severity of non-alcoholic fatty liver disease are underestimated in clinical practice: impact of a dedicated screening approach at a large university teaching hospital. Diabet Med 2018; 35: 89-98

[29] de Lédinghen V, Vergniol J, Gonzalez C et al. Screening for liver fibrosis by using FibroScan ${ }^{\circledR}$ and FibroTest in patients with diabetes. Dig Liver Dis 2012; 44: 413-418

[30] Elkabbany ZA, Elbarbary NS, Ismail EA et al. Transient elastography as a noninvasive assessment tool for hepatopathies of different etiology in pediatric type 1 diabetes mellitus. J Diabetes Complications 2017; 31: 186-194

[31] Williamson RM, Price JF, Glancy S et al. Prevalence of and risk factors for hepatic steatosis and nonalcoholic fatty liver disease in people with type 2 diabetes: The Edinburgh type 2 diabetes study. Diabetes Care 2011; 34: 1139-1344

[32] Sterling RK, Lissen E, Clumeck $N$ et al. Development of a simple noninvasive index to predict significant fibrosis in patients with HIV/ HCV coinfection. Hepatology 2006; 43: 1317-1325
[33] Xiao G, Zhu S, Xiao X et al. Comparison of laboratory tests, ultrasound, or magnetic resonance elastography to detect fibrosis in patients with nonalcoholic fatty liver disease: A meta-analysis. Hepatology 2017; 66: 1486-1501

[34] Sun W, Cui H, Li N et al. Comparison of FIB-4 index, NAFLD fibrosis score and BARD score for prediction of advanced fibrosis in adult patients with non-alcoholic fatty liver disease: A meta-analysis study. Hepatol Res 2016; 46: 862-870

[35] Sarvazyan AP, Rudenko OV, Swanson SD et al. Shear wave elasticity imaging: A new ultrasonic technology of medical diagnostics. Ultrasound Med Biol 1998; 24: 1419-1435

[36] Friedrich-Rust M, Nierhoff ], Lupsor M et al. Performance of Acoustic Radiation Force Impulse imaging for the staging of liver fibrosis: A pooled meta-analysis. J Viral Hepat 2012; 19: 212-219 